\title{
Social Network Analysis of a Chimpanzee (Pan troglodytes) Group in Captivity Following the Integration of a New Adult Member
}

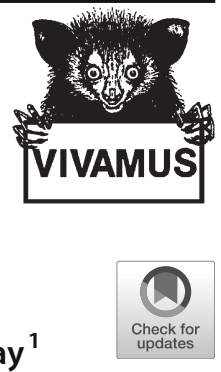

\author{
Sergio Díaz ${ }^{1} \cdot$ Lindsay Murray $^{1} \cdot$ Sam G. B. Roberts $^{2} \cdot$ Paul Rodway $^{1}$ \\ Received: 19 December 2019 / Accepted: 20 August 2020 / Published online: 10 October 2020 \\ (C) The Author(s) 2020
}

\begin{abstract}
Management of primates in captivity often presents the challenge of introducing new individuals into a group, and research investigating the stability of the social network in the medium term after the introduction can help inform management decisions. We investigated the behavior of a group of chimpanzees (Pan troglodytes) housed at Chester Zoo, UK over 12 months (divided into three periods of 4 months) following the introduction of a new adult female. We recorded grooming, proximity, other affiliative behaviors, and agonistic behaviors and used social network analysis to investigate the stability, reciprocity, and structure of the group, to examine the effect of rearing history on grooming network position and the role of sex in agonistic behavior. Both the grooming and agonistic networks correlated across all three periods, while affiliative networks correlated only between periods 2 and 3. Males had significantly higher out-degree centrality in agonistic behaviors than females, indicating that they carried out agonistic behaviors more often than females. There was no significant difference in centrality between hand-reared and mother-reared chimpanzees. Overall, the group structure was stable and cohesive during the first year after the introduction of the new female, suggesting that this change did not destabilize the group. Our findings highlight the utility of social network analysis in the study of primate sociality in captivity, and how it can be used to better understand primate behavior following the integration of new individuals.
\end{abstract}

Keywords Association · Chimpanzees · Grooming · Management · Rearing · Social network analysis $\cdot$ Sex

Handling Editor: Joanna M. Setchell

Sergio Díaz

s.gonzalezdiaz@chester.ac.uk

1 University of Chester, Chester, Cheshire, UK

2 Liverpool John Moores University, Liverpool, Merseyside L3 5UG, UK 


\section{Introduction}

Captive environments offer opportunities for researchers to study and understand primate behavior, but they often also present numerous challenges (Hosey 2005). A common challenge in captive management of primates is the need to integrate new members into an established group. Research on new introductions in captivity is limited and often focuses on how these events inform management decisions (Brent et al. 1997). However, research investigating how new introductions affect the animals themselves in terms of group dynamics and social relationships is scarce.

Primate relationships are well known for being stable over time (Koski et al. 2012), but group changes can potentially alter these relationships, disrupting the group stability (Flack et al. 2006). The introduction of new individuals can alter group dynamics by creating new connections that can disrupt the already established structure of the group. For example, a study on Sulawesi crested macaques (Macaca nigra) found that the introduction of a new male led to a temporary increase in grooming among females of the group, although the grooming network reverted back to normal in the following ten weeks (Cowl et al. 2020). In addition to studying grooming networks, agonistic networks may be good indicators of disruptions after an introduction. For instance, integrations of adult male rhesus macaques (Macaca mulatta) are considered successful if the male remains in the group for 4 weeks without significant behavioral problems (e.g., severe aggression to or from the females in the group; Rox et al. 2019).

Studying the stability of a group after an introduction requires a careful consideration of other factors that can affect the position of individuals in the social network, such as early social history (Suomi 1997), as these factors might be especially relevant during periods in which new social ties are being formed. Rhesus macaques with a history of maternal deprivation show decreased social competence (Kempes et al. 2008) and often fail to reconcile after conflict (Kempes et al. 2009). Common marmosets (Callithrix jacchus) that received reduced early care showed increased signs of stress and decreased social play later in life (Dettling et al. 2002). Social deprivation also has negative effects in adult chimpanzees (Freeman et al. 2016; Murray 1998) and presents an additional challenge in the integration of gorillas (Gorilla gorilla gorilla) in captivity (Burks et al. 2001). Similarly, as agonistic behaviors are particularly relevant as indicators of group stability after introductions (Rox et al. 2019), it is important to consider the possible sex differences in aggression that some primate species are known to exhibit (Bernstein and Ehardt 1985; Fedigan and Baxter 1984; Kulik et al. 2015; Muller and Mitani 2005). Male capuchin monkeys (Sapajus apella), for example, display more frequent agonistic behaviors toward new individuals than females, usually shortly after the introduction but sometimes several days later (Cooper et al. 2001).

Social network analysis is a useful tool to investigate aspects of primate group structure such as reciprocity and stability over time and can also be used to inform the management of animals in captivity (Rose and Croft 2015). For example, high grooming reciprocity is a good predictor of lower aggressive outbreaks in rhesus macaques (McCowan et al. 2008). Similarly, grooming reciprocity is associated with decreased aggression in male Tibetan macaques (Macaca thibetana: Xia et al. 2013), while females tend to show higher reciprocity with other females of adjacent ranks (Xia et al. 2012). Grooming networks are known to be highly stable in rhesus macaques (Massen and Sterck 2013) and female baboons (Papio sp.: Silk et al. 2012), with some 
research showing that wild baboon groups remain stable after group changes, showing only a limited reduction in grooming degree in the first month after an adult leaves the group (Franz et al. 2015).

Chimpanzees are an important species to focus on for the measurement of response to change in the medium term because they are a highly social species that live in multimale, multifemale groups with high fission-fusion dynamics (Lehmann et al. 2007). Further, in the wild female chimpanzees leave their natal community to join neighboring communities (Foerster et al. 2015; Lehman and Boesch, 2009; McCarthy et al. 2018). For resident females, the immigrant females provide more competition for feeding, whereas for resident males, immigrant females provide new opportunities for mating (Kahlenberg et al. 2008a). Female immigrants receive higher levels of aggression than resident females (Kahlenberg et al. 2008b; Pusey et al. 2008), hold lower dominance ranks, and have higher levels of physiological stress, as measured by cortisol (Kahlenberg et al. 2008a). Immigrant females form strong associations with adult males, which intervene to reduce the amount of aggression they receive (Kahlenberg et al. 2008a). Given that immigrant females have a large effect on patterns of sociality in the wild (Kahlenberg et al. 2008a, 2008b; Pusey et al. 2008), it is important to examine the impact of new adult females being introduced into a captive group. Thus, the aim of this study is to examine how the introduction of a new adult female into a captive group affects the stability of the group over the medium term, while also examining two other factors that affect patterns of sociality: early life history and sex.

Chimpanzees in captivity show strong group cohesion and distinct social roles, where each individual contributes to the overall group structure in a different way (Funkhouser et al. 2018; Kanngiesser et al. 2011). Group size has been found to relate to differences in personalities (Murray 1998), with those living in larger groups displaying higher levels of traits associated with positive characteristics - including sociability, gentleness, and intelligence - in contrast to those housed in pairs or trios. Captive groups of chimpanzees are flexible and show stable structures during changes of environment (Koyama and Aureli 2019) and partial stability during changes in dominance (Koyama et al. 2017). The effects of rearing history in adult socialization are well studied in chimpanzees. Chimpanzees that have experienced deprived maternal contact and decreased social interactions manifest personality differences, including higher eccentricity (Murray 1998), and show reduced grooming later in life (Freeman and Ross 2014; Kalcher-Sommersguter et al. 2015). Although chimpanzees with different origins do not show significant differences in grooming centrality or network position in captivity (Levé et al. 2016; Rodrigues and Boeving 2019), group density is significantly affected by the removal of wild-born chimpanzees but not captive-born chimpanzees in simulated models (Levé et al. 2016). Sex differences in chimpanzee aggression are also well documented. Males initiate aggression more often than females (Muller 2002), although females that recently immigrated into the group tend to receive more aggression from females than from males (Kahlenberg et al. 2008a).

In this study, we analyzed social networks based on proximity, grooming, other affiliative behaviors, and agonistic behaviors of the chimpanzee group at Chester Zoo, during the first year after the introduction of a new adult female. Studies often focus on grooming as the only affiliative behavior (Koyama et al. 2017; Levé et al. 2016) or include grooming in the affiliative network (Clark 2011), although some 
studies complement these with proximity networks (Clark 2011; Funkhouser et al. 2018). We investigated proximity, grooming, and other affiliative behaviors (embrace, social play, begging, sharing food, and sexual contact) separately to obtain a more detailed picture of patterns of affiliation in the group. We had three objectives: 1) to investigate the stability, reciprocity, and cohesion of the social networks for grooming and affiliative and agonistic behaviors after the introduction of a new female in the group; 2) to investigate whether chimpanzees differed in their grooming centralities based on their early life histories; and 3) to test whether males and females differed in agonistic behavior.

\section{Methods}

\section{Study Subjects and Site}

The study group consisted of 19 chimpanzees, 7 males and 12 females, housed at Chester Zoo, UK. All ages and kin relations of the chimpanzees are known from zoo records; seven were hand-reared and the rest mother-reared (Table I); reproduction was controlled with reproductive implants during the study.

The chimpanzee group had access to a $143 \mathrm{~m}^{2}, 12 \mathrm{~m}$ high round indoor enclosure, with off-show back beds, and a $2000 \mathrm{~m}^{2}$ outdoor area which they used during the day

Table I Chimpanzee subjects in a study of social networks at Chester Zoo, UK, 2017

\begin{tabular}{lllll}
\hline Name & Sex & Age $^{\mathrm{a}}$ & Hours observed & Rearing history \\
\hline Carlos & M & 12 & 15 & Mother-reared \\
Eric & M & 14 & 17 & Mother-reared \\
Dylan & M & 30 & 15 & Mother-reared \\
Friday & M & 41 & 16.7 & Hand-reared \\
Nicky & M & 48 & 15.7 & Hand-reared \\
Wilson & M & 49 & 15.7 & Hand-reared \\
Boris & M & 51 & 15.7 & Hand-reared \\
Tina & F & 8 & 15 & Mother-reared \\
Patti & F & 20 & 15.7 & Mother-reared \\
Chrissie & F & 21 & 16.3 & Mother-reared \\
Vila & F & 22 & 17 & Mother-reared \\
Zee Zee & F & 23 & 15.7 & Mother-reared \\
Layla & F & 25 & 15 & Mother-reared \\
Alice & F & 26 & 15.3 & Mother-reared \\
Sally & F & 29 & 15.7 & Mother-reared \\
Sarah & F & 31 & 15.3 & Mother-reared \\
Mandy & F & 40 & 17 & Hand-reared \\
Farthing & F & 42 & 16 & Mother-reared \\
Rosie & F & 44 & 16.7 & Hand-reared \\
\hline A & F & & & \\
\hline
\end{tabular}

a Age at beginning of study in January 2017 
(Koyama et al. 2017). The indoor enclosure contained a $9 \mathrm{~m}$ high metal structure with wooden platforms, nets, and ropes as well as nets around the walls and ceiling. The outdoor enclosure contained vertical wooden poles connected with ropes as well as a variety of bushes and grass on the ground. Water was freely available at all times, and food including fruit, vegetables, and primate pellets was provided two to three times a day. Hay was provided as nesting material every morning.

All the chimpanzees formed a single, well-established group. Two chimpanzees were born outside the group: Boris is wild-born and was integrated into the group in 1969 and Farthing was born in a different zoo and was integrated in 1984. No other chimpanzees had been introduced since 1984 and all other individuals were born at Chester Zoo. Vila arrived at the zoo in August 2015 with another adult female, Kiki, who subsequently died in October 2016. Vila was introduced gradually to the group in the off-show area under careful supervision by the keeper team before our study. We investigated the group structure from the moment when Vila was let into the indoor and outdoor zones to associate freely with all group members in January 2017.

\section{Data Collection}

We observed the group for $301 \mathrm{~h}$ from January 2017 to December 2017. Observation sessions usually lasted $4 \mathrm{~h}$, between $00: 10 \mathrm{~h}$ and 15:00 h, during regular zoo opening times. Data collection consisted of 20-min sessions using 1-min instantaneous sampling to record grooming behaviors from or directed to the focal animal and individuals within arm's reach of the focal animal. We recorded all social interactions of all individuals ad libitum (Altmann 1974; Clark 2011). We defined behaviors using Clark's (2011) ethogram (Appendix 1). We chose focal individuals opportunistically from those that were clearly visible, and balanced observations to observe all individuals for a similar amount of time. We calculated interobserver reliability between the main observer and two additional observers for state behaviors during the first month of data collection (Cohen's kappa $\kappa=0.83$ ). We shared monthly reports of the observations of grooming, affiliative and agonistic behaviors, including sociograms and centrality measures, with the primate team at Chester Zoo.

\section{Social Network Measures}

We created four separate networks to analyze different aspects of the group structure: proximity, grooming, other affiliative behaviors, and agonistic networks. Grooming is widely used as an indicator of chimpanzee relationships (Koyama et al. 2017; Levé et al. 2016) and proximity is often used in addition to grooming in social network analyses (Funkhouser et al. 2018; Kalcher-Sommersguter et al. 2015; Schel et al. 2013). Agonistic behaviors (Appendix 1) are commonly reported in management studies, particularly during integrations (Brent et al. 1997; Schel et al. 2013). Other affiliative behaviors are also commonly reported in social network analyses (Clark 2011; Funkhouser et al. 2018) and the behaviors included in this affiliative network were embrace, social play, begging, sharing food, and sexual contact (Appendix 1). We did not include grooming or proximity in the affiliative behaviors network, because we report these separately. Each network had 19 rows and 19 columns, representing the 19 total focal chimpanzees, with a total of 342 dyads. 
We used simple ratio indices ranging $0-1$ to quantify the amount of time spent together or the amount of interaction between individuals while accounting for different observation times. We used these indices to create an undirected (symmetrical) proximity matrix and a directed (asymmetrical) grooming matrix (Whitehead and James 2015). We used half-weight ratio indices, also ranging $0-1$, as a more conservative estimate of association to create directed (asymmetrical) matrices for affiliative and agonistic interactions (Farine and Whitehead 2015).

We calculated in-degree (mean value of interactions received) and out-degree (mean value of interactions given) to assess how well connected each individual was (Rose and Croft 2015). We also calculated betweenness centrality (the number of times the focal is in the shortest path connecting two other nodes) to determine which individuals are important in keeping the group connected (Rose and Croft 2015). Degree is useful to measure which individuals have strong direct connections in the network, while betweenness allows the identification of individuals that play an important role in connecting isolated members of the group (Kanngiesser et al. 2011; Koyama and Aureli 2019). Both measures work well with weighted data in both directed and undirected networks (Borgatti et al. 2013). We calculated all centrality measures using UciNet 6.627 (Borgatti et al. 2002).

\section{Data Analysis}

Network Stability We explored changes in the group structure by dividing the 12mo data collection period into three time periods of 4 mo each: January-April, May-August, and September-December. Studies have used this approach with this group of chimpanzees to examine network stability over time (Koyama et al. 2017). We calculated Quadratic Assignment Procedure (QAP) correlations in R v. 3.6.1 (Core Team 2019) with the interface RStudio 3.5.1 (RStudio Team 2015) using the package sna to test the consistency of grooming, affiliative and agonistic behaviors across the three time periods. This procedure correlates two matrices by modifying them into two columns and calculating Pearson's $r$ between them. It creates a set number of random permutations with the rows and columns of the matrices to create a distribution to compare with the correlation obtained to calculate the significance of the correlation. The $P$ value is calculated by determining the proportion of times that the random correlations are larger than the observed correlation. All analyses used 5000 permutations and an $\alpha$ value of 0.05 .

Network Reciprocity and Subgroup Detection We analyzed network reciprocity in grooming, agonistic behaviors, and affiliative behaviors using Mantel Z-tests with the ape package in R v. 3.6.1 (Core Team 2019) using RStudio 3.5.1 (RStudio Team 2015). Mantel $Z$-tests are a permutation-based procedure used to detect reciprocity in behaviors, obtaining a matrix correlation coefficient by correlating the nondiagonal elements of two matrices (Hemelrijk 1990).

We used hierarchical cluster analysis in UciNet 6.627 (Borgatti et al. 2002), using the average between pairs method, to create a dendrogram and detect subgroups in the proximity matrix, and in the symmetrized grooming, agonistic, and affiliative matrices. This algorithm detects the strongest similarity between two elements (e.g., the two 
chimpanzees that have spent the most time in close proximity) and clusters them together. The software repeats this step until a single cluster represents the whole group, and then provides Q coefficients to measure how well-defined the subgroups are. Q coefficients with values of 0.3 or more indicate good divisions of the network into subgroups (Newman 2004).

\section{Sex and Rearing History Differences}

We calculated node-level permutation $t$-tests using the coin package in R v. 3.6.1 (Core Team 2019) using RStudio 3.5.1 (RStudio Team 2015) to test for differences in the centrality indices of the grooming matrix between hand-reared and mother-reared chimpanzees, and for differences in the centrality indices of the agonistic matrix between males and females using an $\alpha$ value of 0.05 . We used the package effsize to calculate the effect size using Cohen's $d$. We also used a permutation-based ANOVA (symmetry test) to further investigate differences across the three periods in out-degree (the mean value of agonistic behavior by the focal individual directed at other individuals) to study changes in agonistic behavior over time. We used post hoc tests with adjusted $P$ values to control the false discovery rate (Benjamini and Hochberg 1995; Benjamini and Yekutieli 2001).

\section{Ethical Note}

The study was approved by the Ethics Committee of the School of Psychology at the University of Chester and the Research Committee of Chester Zoo. The study required only observational data; no manipulation of the animals or their environment was needed, and we observed animals during their normal display hours at the zoo. Authors declare that there is no conflict of interest.

Data Availability The datasets used and analyzed during the current study are available from the corresponding author on reasonable request.

\section{Results}

\section{Frequency of Behaviors}

Each chimpanzee was involved in a mean of 0.79 (SD 0.63) affiliative interactions per hour, and in a mean of 0.50 (SD 0.77) agonistic interactions per hour. Over the three time periods, chimpanzees spent a mean of $12.1 \%$ of their time grooming (SD $5.8 \%$ ). Vila, the newly integrated chimpanzee, groomed reciprocally with Eric and received grooming from Rosie (Fig. 1). Vila displayed affiliative ties to Tina, the youngest female in the group, and Dylan, the alpha male, who was central to both the affiliative and grooming networks (Fig. 2). However, Vila was unconnected in 


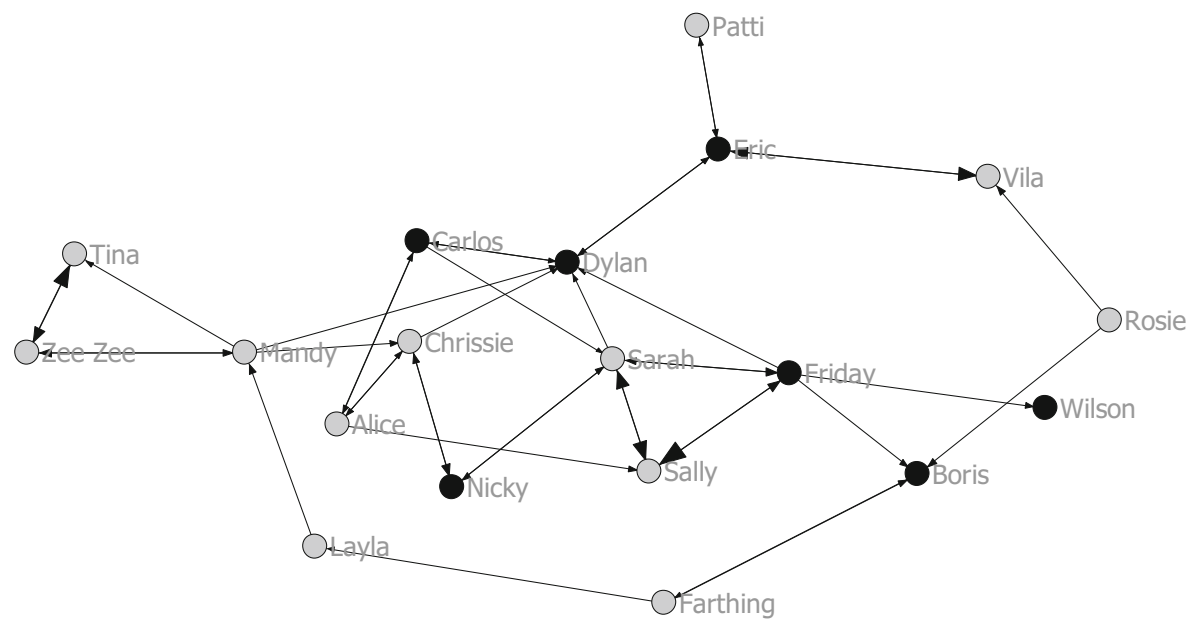

Fig. 1 Grooming network over 12 mo, showing strong grooming ties (one SD above the mean) for the chimpanzee group at Chester Zoo, UK (2017). Females are displayed in gray and males in black; arrowheads represent direction and strength.

the agonistic network because she did not receive, or give, strong agonistic behavior (Fig. 3).

\section{Correlations Between Behaviors}

For the full year, we found a significant positive QAP correlation between grooming and proximity $(r=0.595, P<0.001, N=342$ dyads) but the correlations between grooming and affiliative behaviors $(r=0.119, P=0.061, N=342$

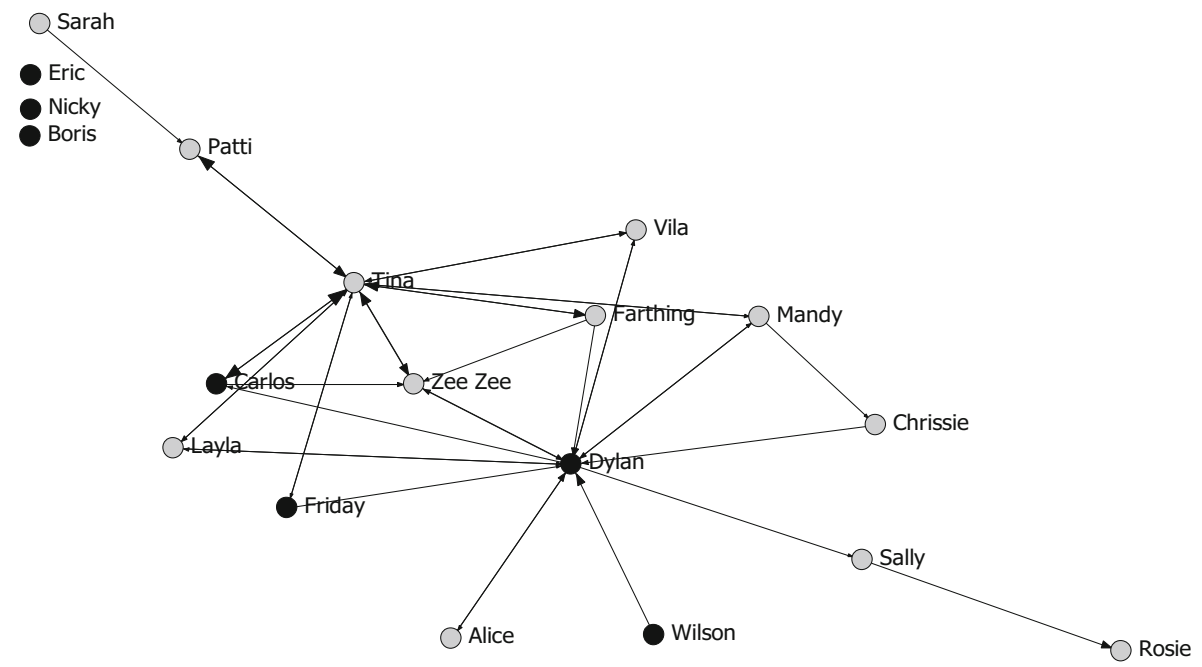

Fig. 2 Affiliative network over 12 mo, showing strong affiliative ties (one SD above the mean) for the chimpanzee group at Chester Zoo, UK (2017). Females are displayed in gray and males in black; arrowheads represent direction and strength. 


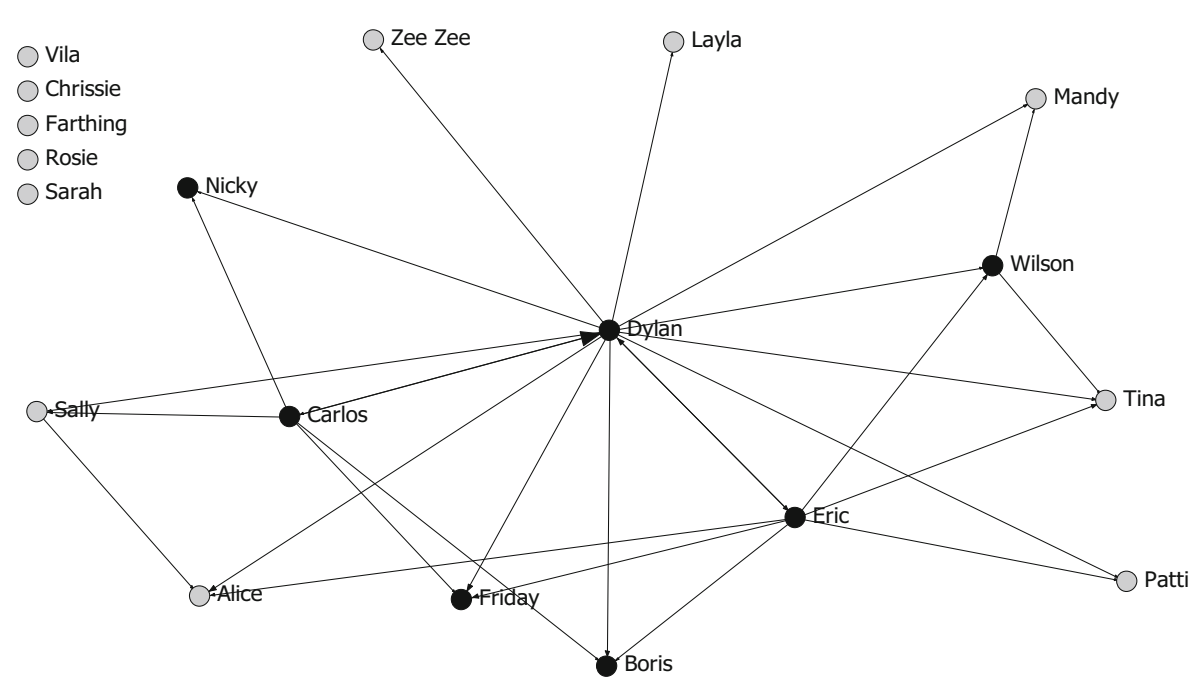

Fig. 3 Agonistic network over $12 \mathrm{mo}$, showing strong agonistic ties (one SD above the mean) for the chimpanzee group at Chester Zoo, UK (2017). Females are displayed in gray and males in black; arrowheads represent direction and strength.

dyads) and between grooming and agonistic behaviors $(r=0.064, P=0.130, N=$ 342 dyads) were not statistically significant.

\section{Correlations Between Time Periods}

We found significant positive QAP correlations for grooming between the first and second periods, and between the second and third periods (Table II). For affiliative behaviors, we did not find a significant correlation between the first and second periods, but there was a significant positive correlation between the second and third periods. Finally, for agonistic behaviors, the QAP analysis found significant positive correlations between the first and second period, and between the second and third periods.

Table II Quadratic Assignment Procedure correlation coefficients between three time periods for grooming, affiliative, and agonistic behaviors for the chimpanzee group at Chester Zoo, UK (2017)

\begin{tabular}{llll}
\hline & Grooming & Affiliative & Agonistic \\
\hline Periods 1-2 & $0.316^{\mathrm{a}}$ & 0.081 & $0.319^{\mathrm{a}}$ \\
Periods 2-3 & $0.415^{\mathrm{a}}$ & $0.391^{\mathrm{a}}$ & $0.411^{\mathrm{a}}$ \\
\hline
\end{tabular}

$N=342$ dyads

${ }^{a}$ Indicates correlations significant at $P<0.01$ 


\section{Network Reciprocity}

We found significant reciprocity in grooming (Mantel $Z$-test $P<0.001$ ), agonistic behaviors $(P<0.044)$, and affiliative behaviors $(P<0.001)$, indicating that chimpanzees tended to reciprocate interactions.

\section{Subgroup Detection}

Hierarchical cluster analysis did not detect a good division into subgroups in grooming $(Q=0.228)$, although the adequacy of grooming clusters was close to the cutoff of $Q>$ 0.03 . There were no good subgroups in proximity $(Q=0.067)$, affiliative behaviors $(Q$ $=0.142)$, or agonistic behaviors $(Q=0.011)$.

\section{Rearing History}

We did not find significant differences between hand-reared and mother-reared chimpanzees in out-degree (permutation $t$-test $Z=-1.18, P=0.251, d=0.59$; Table III), indegree $(Z=-1.40, P=0.167, d=0.71$; Table III) or betweenness centrality $(Z=-1.07$, $P=0.292, d=0.53$; Table III), indicating that chimpanzees with different early life histories did not differ in the amount of grooming partners they had, or in their importance as interconnecting individuals between other members of the group (Table III).

\section{Sex Differences}

The out-degree for agonistic behaviors was significantly higher in males than in females $(Z=-2.29, P=0.018, d=1.26$; Table IV), indicating that males directed more agonistic behaviors at other individuals than females did, but we found no sex differences in in-degree $(Z=-1.74, P=0.077, d=0.88)$ or betweenness centrality $(Z=-0.67, P=0.519, d=0.31)$ (Table IV), indicating that males and females did not differ in the amount of agonistic behavior received or directed to other individuals. We found a significant effect of time period on female agonistic out-degree (symmetry test $T=2.57, P=0.027$ ) (Table V), but pairwise comparisons with adjusted $P$ values revealed no significant differences between periods (Table VI). We also found a significant effect of time period of male out-degree in agonistic behaviors $(T=2.92$, $P=0.01$ ), with pairwise comparisons revealing that male out-degree in agonistic behaviors was higher in period 3 than in periods 2 or 1 (Table VI).

Table III Mean (and standard deviation) grooming centrality for hand-reared and mother-reared chimpanzees at Chester Zoo, UK (2017)

\begin{tabular}{lll}
\hline & Hand-reared & Mother-reared \\
\hline Out-degree & $0.10(0.07)$ & $0.13(0.04)$ \\
In-degree & $0.10(0.04)$ & $0.13(0.04)$ \\
Betweenness & $3.21(2.55)$ & $4.29(4.72)$ \\
\hline
\end{tabular}


Table IV Mean (and standard deviation) agonistic centrality by sex for the chimpanzee group at Chester Zoo, UK (2017)

\begin{tabular}{lll}
\hline & Males & Females \\
\hline Out-degree & $0.62(0.66)$ & $0.112(0.11)$ \\
In-degree & $0.40(0.23)$ & $0.241(0.15)$ \\
Betweenness & $17.07(15.47)$ & $12.54(14.02)$ \\
\hline
\end{tabular}

\section{Discussion}

We found that the grooming network and agonistic network were stable between the three 4-mo study periods in this captive group of chimpanzees after the integration of a new adult female into the group. However, the network using other affiliative behaviors was stable only between the second and third periods. The three networks showed significant reciprocity, but we detected no subgroups in the group. There were no differences in grooming between hand-reared and mother-reared chimpanzees. Finally, males performed significantly more agonistic behaviors than females with a large effect size, although there was no difference in in-degree, meaning that neither females nor males were more likely to be targeted in aggression, and betweenness centrality, which indicates that neither females not males were more likely to display agonistic behaviors toward less connected individuals. Moreover, male agonistic behavior increased significantly during the third 4-mo period of the year.

The grooming network correlated moderately across the three 4-mo time periods of the study, indicating that it was moderately stable during the first year after the integration of a new group member. Primate networks are highly stable over time (Franz et al. 2015; Massen and Sterck 2013) and our results indicate that chimpanzee grooming networks retain some stability during periods where the group undergoes changes, supporting previous findings that indicate that grooming networks in captive chimpanzees correlate between periods of dominance uncertainty (Koyama et al. 2017). The agonistic network of the group also showed moderate correlations across the three time periods. These results contrast with those found in a study showing that agonistic behavior reduced in the year following the integration of two chimpanzee groups (Schel et al. 2013). This difference in results can be explained by the fact that integrating two groups is a much bigger disruptor than integrating a single female, and it is possible that agonistic behavior increased particularly among male chimpanzees, as males show high intergroup aggression (Muller and Mitani 2005). Our results showed

Table V Mean (and standard deviation) out-degree by sex during three 4-mo periods for the chimpanzee group at Chester Zoo, UK (2017)

\begin{tabular}{lll}
\hline & Males & Females \\
\hline Period 1 & $3.29(3.45)$ & $1.08(1.16)$ \\
Period 2 & $4.14(5.43)$ & $0.92(1.16)$ \\
Period 3 & $7.86(6.20)$ & $2.58(2.61)$ \\
\hline
\end{tabular}


Table VI Adjusted $P$ values for pairwise comparisons of out-degree in agonistic behavior between three 4-mo time periods for the chimpanzee group at Chester Zoo, UK (2017)

\begin{tabular}{llc}
\hline & Females & Males \\
\hline Periods 1 and 2 & 0.637 & 0.355 \\
Periods 1 and 3 & 0.142 & $0.046^{\mathrm{a}}$ \\
Periods 2 and 3 & 0.087 & $0.046^{\mathrm{a}}$ \\
\hline
\end{tabular}

${ }^{a}$ Indicates significant corrected $P$ values

only a moderate correlation between the second and third time periods for affiliative behaviors, which could be due to initial changes in the affiliative network in the first period, followed by more settled structure in the next two periods. However, the affiliative network in this study includes diverse behaviors such as play and sexual interactions and an alternative explanation for the lack of temporal stability between the first and second periods is that these affiliative behaviors serve different functions during these two periods. These results match those found in a study on dyadic interactions between newly introduced chimpanzees that showed how grooming could be observed early in the introduction but other affiliative behaviors such as play would appear later in the process (Brent et al. 1997). The lack of a significant correlation between the grooming network and the affiliative network highlights the need to consider grooming behaviors separately from other interactions and indicates that this approach might offer a more nuanced picture of primate groups.

The chimpanzee group at Chester Zoo shows high grooming reciprocity, with females reciprocating grooming even during periods of dominance instability (Koyama et al. 2017), and our results indicate that the group also shows reciprocity in other affiliative behaviors. Higher grooming reciprocity is associated with lower aggression rates in macaques (Macaca nemestrina: Flack et al. 2006; Macaca sylvanus: McCowan et al. 2008), which may reflect a positive overall group dynamic and could indicate that that the integration of the new chimpanzee in the group is going well. Our results contrast with a study that did not find reciprocity in grooming in a sample of seven chimpanzees housed in a sanctuary (Funkhouser et al. 2018). This difference may have been because grooming reciprocity is higher among related than among unrelated chimpanzees (Fedurek and Dunbar 2009). The sanctuary animals were genetically unrelated (Funkhouser et al. 2018), whereas the chimpanzee group at Chester Zoo has several maternally related individuals (Koyama et al. 2017). Our results support previous findings of reciprocity in agonistic interactions (Funkhouser et al. 2018). These data indicate that although some individuals are more central in the agonistic network (i.e., Dylan and Carlos), they do not dominate agonistic behaviors. However, it is important to consider that observations of agonistic behavior are often limited in captivity, particularly in big groups or in situations with limited space to avoid interactions (Videan and Fritz 2007).

Although we found distinct preferences in the way each individual interacts with others, hierarchical cluster analysis did not detect subgroups. This finding supports previous findings that also did not find significant subgroups in captive chimpanzees (Clark 2011; Funkhouser et al. 2018), although the authors stressed the need to be cautious when interpreting a lack of subgroups, as groups of chimpanzees might 
change too quickly to be reflected when studied over a period of several months (Clark 2011). Studies of chimpanzees (Brent et al. 1997) and of macaques (Cowl et al. 2020; Rox et al. 2019) show that successful integrations might disrupt the group networks in the first four weeks. Our findings indicate that, in the medium term after the integration, the overall group structure was moderately stable, reciprocal, and cohesive, showing that the group was well adapted to the new arrival and did not experience strong changes during this period. The position of Vila, the newly introduced female, in the grooming and affiliative network showed strong ties to central individuals such as Dylan, and her position in the agonistic network indicated that she did not commonly receive or give aggression. Thus, while immigrant females in wild groups have significant effects on patterns of female-female and male-female sociality and aggression (Kahlenberg et al. 2008a) the introduction of a single female did not appear to have a disruptive effect on the social network of a captive group. One important difference may be that whereas in wild groups there are multiple immigrant females, creating tension between resident males, resident females and immigrant females (Kahlenberg et al. 2008a, b; Pusey et al. 2008), our study focused only on a single new female immigrant into an otherwise stable group.

We found no significant difference in grooming centrality between hand-reared and mother-reared chimpanzees. The two hand-reared females in the group (Mandy and Rosie) are both well connected to their daughters, and the increased reciprocity between kin might contribute to their centrality in the grooming network. Deprivation of social maternal contact during early life reduces grooming activity throughout adult life in chimpanzees (Freeman and Ross 2014; Kalcher-Sommersguter et al. 2013), while research with rhesus macaques has shown that it is associated with less reconciliation after a fight (Kempes et al. 2008, 2009), although the effect on network position is still unclear. More research into the mediating effects of family relationships and, potentially, resocialization efforts in sanctuary settings, could help build a more detailed understanding of the long-lasting impact of early life on network position and social role.

Males showed significantly higher out-degree in agonistic behavior than females with a large effect size, which is to be expected in chimpanzees, as males tend to show more aggression than females and in a wider range of contexts, from fights for dominance to food competition (Muller 2002). In particular, three individuals held central positions in the agonistic network: Dylan, who has been identified as the dominant individual since 2002 (Koyama et al. 2017), and two young males that often display and fight with him, Carlos and Eric. In the wild, males may protect new females from aggression by resident females (Kahlenberg et al. 2008a). Nevertheless, we found no significant sex difference in in-degree or betweenness centrality, indicating that agonistic behaviors were not directed preferentially towards females or other males. In contrast to the high levels of aggression received by immigrant females in the wild (Kahlenberg et al. 2008a), Vila, the new female chimpanzee, did not receive aboveaverage agonistic behavior from other members of the group, but did show strong grooming ties to central males in the group (Dylan during the first period and Eric throughout the year), which might have helped her avoid agonistic interaction with other females. While there are important differences between patterns of sociality in captivity and the wild, for example, in the fission-fusion social structure (Lehmann et al. 2007), our results suggest that new adult females introduced into captive groups may follow the strategy seen in wild chimpanzees of forming stronger associations with 
males than females (Kahlenberg et al. 2008a). Further research could examine whether this is a consistent finding when new adult females are introduced in captivity, whether males intervene to prevent the new female receiving aggression in captive settings as they do in the wild (Kahlenberg et al. 2008a) and whether the social network of wild chimpanzee groups also shows stability when new adult females enter the group.

Interestingly, male agonistic behavior significantly increased during the third period of the study. Although space restriction due to bad weather was more common during the last period of the study, this is unlikely to be a determining factor in the increase of male agonistic behavior, as studies found no differences in mutual grooming and agonistic behavior in the same group during periods of space restriction (Koyama and Aureli 2019). Instead, this increase in male agonistic behavior is likely to indicate intensification of the conflict between the dominant male and the two young males that were beginning to challenge his dominance.

We must consider several limitations when interpreting our findings. First, the affiliative network groups together social play, begging, sharing food, and sexual behaviors. We included this affiliative network to give a more comprehensive picture of affiliation in the group than relying only on grooming. However, grouping behaviors can present problems when the behaviors might have different functions and may explain why some researchers focus solely on grooming networks (Funkhouser et al. 2018; Kalcher-Sommersgutter et al. 2015; Koyama et al. 2017; Levé et al. 2016; Schel et al. 2013). Considering additional affiliative behaviors separately instead of grouping them may be a valuable approach in future. Future research could also consider the use of multilayered approaches to the study of affiliation, which allow researchers to integrate and examine multiple indices of social interactions (Silk et al. 2013, 2018; Smith-Aguilar et al. 2019).

A second limitation is that the study examined the development of the group structure after the integration of a new female but did not use data prior to the integration. We cannot assess changes in the network as a consequence of the arrival of the new chimpanzee. Instead, we focused on the medium-term stability of the group structure. Similarly, we do not include information from before Vila had access to the full enclosure, which could be valuable in interpreting her position in the networks and her individual ties.

Despite these limitations, our findings extend previous work on social networks in primates (Clark 2011; Funkhouse et al. 2018; Koyama and Aureli 2019; Koyama et al. 2017; Massen and Sterck 2013; McCowan et al. 2008; Silk et al. 2012) by examining how a chimpanzee group adapted to the integration of a new adult female, as well as investigating the effects of rearing history and sex in the social network. The group structure proved to be moderately stable and cohesive during the first year after the introduction of the female, suggesting that the integration was successful, although affiliative behaviors were stable only in the second and third periods. Rearing history did not significantly affect grooming centrality. Males were more agonistic than females, particularly during the third period. The new chimpanzee, Vila, formed strong grooming ties to central male individuals in the group and was not strongly connected in the agonistic network, indicating that she was not a common target of agonistic behavior and that she did not direct frequent agonistic behaviors at others. Further research could examine primate social networks before new introductions to better understand changes produced by the arrival. The findings show that chimpanzee groups can adapt well to new integrations and illustrate how social network analysis can be used to understand primate behavior in captivity, potentially helping management decisions. 
Acknowledgements We would like to thank the research team and the primate keepers at Chester Zoo for all their assistance with this research. We would like to thank the editor and two anonymous reviewers for their comments and recommendations on the manuscript.

Author Contributions SD, LM and SR conceived the study. SD developed the methodology, collected and analyzed the data and wrote the manuscript. LM, SR and PR provided editorial advice.

\section{Appendix 1}

Table VII Ethogram modified from Clark (2011), showing sampling methods and affiliative, agonistic, and state behaviors recorded as well as frequency and percentage of each behavior for affiliative and agonistic behaviors

\begin{tabular}{|c|c|c|c|}
\hline $\begin{array}{l}\text { Sampling } \\
\text { method }\end{array}$ & & Description & $\begin{array}{l}\text { Frequency } \\
\text { and } \\
\text { percentage }\end{array}$ \\
\hline \multirow{7}{*}{$\begin{array}{l}\text { Ad libitum } \\
\text { sampling }\end{array}$} & Affiliative & & \\
\hline & Embrace & Embrace or hug another individual & $35(11.5 \%)$ \\
\hline & Social play & $\begin{array}{l}\text { Tussle and chase another individual. May be accompanied by } \\
\text { the "play face" and patting vocalizations }\end{array}$ & $63(20.3 \%)$ \\
\hline & Beg & Offer hand outstretched to another individual to solicit support & $99(32.5 \%)$ \\
\hline & Share food & $\begin{array}{l}\text { Allow another to share the same piece or small pile of food. } \\
\text { Handle food with another or tolerate food being taken }\end{array}$ & $11(3.6 \%)$ \\
\hline & Sexual & $\begin{array}{l}\text { Heterosexual mount that may be followed by thrust and } \\
\text { intromission. Inspect genitals to solicit sexual activity }\end{array}$ & $40(13.1 \%)$ \\
\hline & Other & Other affiliative behaviors not listed above & $58(19 \%)$ \\
\hline \multirow{5}{*}{$\begin{array}{l}\text { Ad libitum } \\
\text { sampling }\end{array}$} & Agonistic & & \\
\hline & $\begin{array}{l}\text { Dominance } \\
\text { mount }\end{array}$ & $\begin{array}{l}\text { Mount another individual in a nonsexual context, } \\
\text { or position rear-end toward another to solicit mounting }\end{array}$ & $38(13.9 \%)$ \\
\hline & $\begin{array}{c}\text { Displace or } \\
\text { supplant }\end{array}$ & $\begin{array}{l}\text { Approach another individual and cause their retreat. } \\
\text { May be related to access to a resource such as food }\end{array}$ & $\begin{array}{l}110 \\
(40.1 \%)\end{array}$ \\
\hline & $\begin{array}{l}\text { Noncontact } \\
\text { threat }\end{array}$ & $\begin{array}{l}\text { Various behaviors including charge and lunge. } \\
\text { May be accompanied by bristling hair. } \\
\text { Display aimed at group, subgroup, or one individual }\end{array}$ & $47(17.2 \%)$ \\
\hline & Attack & Physical aggressive contact such as hit or bite & $79(28.8 \%)$ \\
\hline \multirow{9}{*}{$\begin{array}{l}\text { 1-min focal } \\
\text { scan } \\
\text { sampling }\end{array}$} & $\begin{array}{l}\text { State } \\
\text { behaviors }\end{array}$ & & \\
\hline & Proximity & \multicolumn{2}{|l|}{$\begin{array}{l}\text { Identity of individuals within arm's reach of the focal } \\
\text { individual }\end{array}$} \\
\hline & Feed & \multicolumn{2}{|l|}{$\begin{array}{l}\text { Eat or drink from diet, enclosure substrate, } \\
\text { or food-based enrichment }\end{array}$} \\
\hline & Forage & \multicolumn{2}{|l|}{ Actively search for food } \\
\hline & Locomote & \multicolumn{2}{|l|}{ Move bipedally or quadrupedally } \\
\hline & Explore & \multicolumn{2}{|l|}{$\begin{array}{l}\text { Investigate environment, but not in relation to food. } \\
\text { Vigilant to visitors or keepers }\end{array}$} \\
\hline & Rest & \multicolumn{2}{|l|}{ Rest or sleep in varying postures } \\
\hline & Social & \multicolumn{2}{|l|}{$\begin{array}{l}\text { Agonistic and affiliative behavior. } \\
\text { Specific social interactions recorded separately (see below) }\end{array}$} \\
\hline & Autogrooming & & \\
\hline
\end{tabular}


Table VII (continued)

\begin{tabular}{|c|c|c|}
\hline $\begin{array}{l}\text { Sampling } \\
\text { method }\end{array}$ & Description & $\begin{array}{l}\text { Frequency } \\
\text { and } \\
\text { percentage }\end{array}$ \\
\hline
\end{tabular}

\author{
Pick through own hair, examine skin, \\ and remove dirt and detritus \\ Allogrooming Pick through the hair of another individual, examine skin, \\ and remove dirt and detritus. \\ Behavior may or may not be reciprocated \\ Other \\ Any behavior not listed above
}

Open Access This article is licensed under a Creative Commons Attribution 4.0 International License, which permits use, sharing, adaptation, distribution and reproduction in any medium or format, as long as you give appropriate credit to the original author(s) and the source, provide a link to the Creative Commons licence, and indicate if changes were made. The images or other third party material in this article are included in the article's Creative Commons licence, unless indicated otherwise in a credit line to the material. If material is not included in the article's Creative Commons licence and your intended use is not permitted by statutory regulation or exceeds the permitted use, you will need to obtain permission directly from the copyright holder. To view a copy of this licence, visit http://creativecommons.org/licenses/by/4.0/.

\title{
References
}

Altmann, J. (1974). Observational study of behavior: Sampling methods. Behavior, 49(3), 227-259.

Benjamini, Y., \& Hochberg, Y. (1995). Controlling the false discovery rate: A practical and powerful approach to multiple testing. Journal of the Royal Statistical Society Series B (Methodological), 57(1), 289-300. https://doi.org/10.2307/2346101.

Benjamini, Y., \& Yekutieli, D. (2001). The control of the false discovery rate in multiple testing under dependency. Annals of Statistics, 29(4), 1165-1188. https://doi.org/10.1214/aos/1013699998.

Bernstein, I. S., \& Ehardt, C. L. (1985). Age-sex differences in the expression of agonistic behavior in rhesus monkey (Macaca mulatta) groups. Journal of Comparative Psychology, 99(2), 115-132. https://doi.org/ 10.1037/0735-7036.99.2.115.

Borgatti, S. P., Everett, M. G., \& Freeman, L. C. (2002). Ucinet 6 for Windows: Software for social network analysis. Harvard, MA: Analytic Technologies.

Borgatti, S. P., Everett, M. G., \& Johnson, J. C. (2013). Analyzing social networks. London: SAGE.

Brent, L., Kessel, A. L., \& Barrera, H. (1997). Evaluation of introduction procedures in captive chimpanzees. Zoo Biology, 16(4), 335-342. https://doi.org/10.1002/(SICI)1098-2361(1997)16:4<335::AID-ZOO5>3.0.CO;2-B.

Burks, K. D., Bloomsmith, M. A., Forthman, D. L., \& Maple, T. L. (2001). Managing the socialization of an adult male gorilla (Gorilla gorilla gorilla) with a history of social deprivation. Zoo Biology, 20(5), 347358.

Clark, F. E. (2011). Space to choose: Network analysis of social preferences in a captive chimpanzee community, and implications for management. American Journal of Primatology, 73(8), 748-757. https://doi.org/10.1002/ajp.20903.

Cooper, M. A., Bernstein, I. S., Fragaszy, D. M., \& de Waal, F. B. M. (2001). Integration of new males into four social groups of tufted capuchins (Cebus apella). International Journal of Primatology, 22(4), 663683. https://doi.org/10.1023/A:1010745803740.

Core Team, R. (2019). R: A language and environment for statistical computing. Vienna, Austria: $R$ Foundation for Statistical Computing. https://www.R-project.org/.

Cowl, V. B., Jensen, K., Lea, J. M. D., Walker, S. L., \& Shultz, S. (2020). Sulawesi crested macaque (Macaca nigra) grooming networks are robust to perturbation while individual associations are more labile. International Journal of Primatology, 41(1), 105-128. https://doi.org/10.1007/s10764-020-00139-6.

Dettling, A. C., Feldon, J., \& Pryce, C. R. (2002). Repeated parental deprivation in the infant common marmoset (Callithrix jacchus, primates) and analysis of its effects on early development. Biological Psychiatry, 52(11), 1037-1046. 
Farine, D. R., \& Whitehead, H. (2015). Constructing, conducting and interpreting animal social network analysis. Journal of Animal Ecology, 84(5), 1144-1163. https://doi.org/10.1111/1365-2656.12418.

Fedigan, L. M., \& Baxter, M. J. (1984). Sex differences and social organization in free-ranging spider monkeys (Ateles geoffroyi). Primates, 25(3), 279-294. https://doi.org/10.1007/BF02382267.

Fedurek, P., \& Dunbar, R. I. M. (2009). What does mutual grooming tell us about why chimpanzees groom? Ethology, 115(6), 566-575. https://doi.org/10.1111/j.1439-0310.2009.01637.x.

Flack, J. C., Girvan, M., De Waal, F. B. M., \& Krakauer, D. C. (2006). Policing stabilizes construction of social niches in primates. Nature, 439, 426-429. https://doi.org/10.1038/nature04326.

Foerster, S., McLellan, K., Schroepfer-Walker, K., Murray, C. M., Krupenye, C., et al (2015). Social bonds in the dispersing sex: Partner preferences among adult female chimpanzees. Animal Behaviour, 105, 139152. https://doi.org/10.1016/j.anbehav.2015.04.012.

Franz, M., Altmann, J., \& Alberts, S. C. (2015). Knockouts of high-ranking males have limited impact on baboon social networks. Current Zoology, 61(1), 107-113. https://doi.org/10.1093/czoolo/61.1.107.

Freeman, H. D., \& Ross, S. R. (2014). The impact of atypical early histories on pet or performer chimpanzees. PeerJ, 2, e579. https://doi.org/10.7717/peerj.579.

Freeman, H. D., Weiss, A., \& Ross, S. R. (2016). Atypical early histories predict lower extraversion in captive chimpanzees. Developmental Psychobiology, 58(4), 519-527. https://doi.org/10.1002/dev.21395.

Funkhouser, J. A., Mayhew, J. A., \& Mulcahy, J. B. (2018). Social network and dominance hierarchy analyses at Chimpanzee Sanctuary Northwest. PLoS ONE, 13(2), e0191898. https://doi.org/10.1371/journal.pone.0191898.

Hemelrijk, C. K. (1990). Models of, and tests for, reciprocity, unidirectionality and other social interaction patterns at a group level. Animal Behavior, 39(6), 1013-1029. https://doi.org/10.1016/S0003-3472(05)80775-4.

Hosey, G. R. (2005). How does the zoo environment affect the behavior of captive primates? Applied Animal Behavior Science, 90(2), 107-129. https://doi.org/10.1016/J.APPLANIM.2004.08.015.

Kahlenberg, S. M., Thompson, M. E., Muller, M. N., \& Wrangham, R. W. (2008a). Immigration costs for female chimpanzees and male protection as an immigrant counterstrategy to intrasexual aggression. Animal Behavior, 76(5), 1497-1509. https://doi.org/10.1016/j.anbehav.2008.05.029.

Kahlenberg, S. M., Emery Thompson, M., \& Wrangham, R. W. (2008b). Female Competition over core areas in Pan troglodytes schweinfurthii, Kibale National Park, Uganda. International Journal of Primatology, 29(4), 931-947. https://doi.org/10.1007/s10764-008-9276-3.

Kalcher-Sommersguter, E., Franz-Schaider, C., Crailsheim, K., \& Preuschoft, S. (2013). Social competence of adult chimpanzees (Pan troglodytes) with severe deprivation history: A relational approach. International Journal of Comparative Psychology, 26(2), 135-157.

Kalcher-Sommersguter, E., Preuschoft, S., Franz-Schaider, C., Hemelrijk, C. K., Crailsheim, K., \& Massen, J. J. M. (2015). Early maternal loss affects social integration of chimpanzees throughout their lifetime. Scientific Reports, 5(1), 16439. https://doi.org/10.1038/srep16439.

Kanngiesser, P., Sueur, C., Riedl, K., Grossmann, J., \& Call, J. (2011). Grooming network cohesion and the role of individuals in a captive chimpanzee group. American Journal of Primatology, 73(8), 758-767. https://doi.org/10.1002/ajp.20914.

Kempes, M. M., Gulickx, M. M. C., van Daalen, H. J. C., Louwerse, A. L., \& Sterck, E. H. M. (2008). Social competence is reduced in socially deprived rhesus monkeys (Macaca mulatta). Journal of Comparative Psychology, 122(1), 62-67. https://doi.org/10.1037/0735-7036.122.1.62.

Kempes, M. M., Den Heijer, E., Korteweg, L., Louwerse, A. L., \& Sterck, E. H. M. (2009). Socially deprived rhesus macaques fail to reconcile: Do they not attempt or not accept reconciliation? Animal Behaviour, 78(2), 271-277. https://doi.org/10.1016/j.anbehav.2009.04.013.

Koski, S. E., de Vries, H., van de Kraats, A., \& Sterck, E. H. M. (2012). Stability and Change of Social Relationship Quality in Captive Chimpanzees (Pan troglodytes). International Journal of Primatology, 33(4), 905-921. https://doi.org/10.1007/s10764-012-9623-2

Koyama, N. F., \& Aureli, F. (2019). Social network changes during space restriction in zoo chimpanzees. Primates, 60(3), 203-211. https://doi.org/10.1007/s10329-018-0675-6.

Koyama, N. F., Ronkainen, K., \& Aureli, F. (2017). Durability and flexibility of chimpanzee grooming patterns during a period of dominance instability. American Journal of Primatology, 79(11), e22713. https://doi.org/10.1002/ajp.22713.

Kulik, L., Amici, F., Langos, D., \& Widdig, A. (2015). Sex differences in the development of aggressive behavior in rhesus macaques (Macaca mulatta). International Journal of Primatology, 36(4), 764-789. https://oi.org/10.1007/s10764-015-9853-1.

Lehmann, J., \& Boesch, C. (2009). Sociality of the dispersing sex: The nature of social bonds in West African female chimpanzees, Pan troglodytes. Animal Behaviour, 77(2), 377-387. https://doi.org/10.1016/j. anbehav.2008.09.038. 
Lehmann, J., Korstjens, A. H., \& Dunbar, R. I. M. (2007). Fission-fusion social systems as a strategy for coping with ecological constraints: A primate case. Evolutionary Ecology, 21(5), 613-634.

Levé, M., Sueur, C., Petit, O., Matsuzawa, T., \& Hirata, S. (2016). Social grooming network in captive chimpanzees: Does the wild or captive origin of group members affect sociality? Primates, 57(1), 73-82. https://doi.org/10.1007/s10329-015-0494-y.

Massen, J. J. M., \& Sterck, E. H. M. (2013). Stability and durability of intra- and intersex social bonds of captive rhesus macaques (Macaca mulatta). International Journal of Primatology, 34(4), 770-791. https://doi.org/10.1007/s10764-013-9695-7.

McCarthy, M. S., Lester, J. D., Langergraber, K. E., Stanford, C. B., \& Vigilant, L. (2018). Genetic analysis suggests dispersal among chimpanzees in a fragmented forest landscape in Uganda. American Journal of Primatology, 80(9), e22902. https://doi.org/10.1002/ajp.22902.

McCowan, B., Anderson, K., Heagarty, A., \& Cameron, A. (2008). Utility of social network analysis for primate behavioral management and well-being. Applied Animal Behavior Science, 109(2-4), 396-405.

Muller, M. N. (2002). Agonistic relations among Kanyawara chimpanzees. In C. Boesch, G. Ohmann, \& L. Marchant (Eds.), Behavioural diversity in chimpanzees and bonobos (pp. 112-124). Cambridge: Cambridge University Press.

Muller, M. N., \& Mitani, J. C. (2005). Conflict and cooperation in wild chimpanzees. Advances in the Study of Behavior, 35, 275-331. https://doi.org/10.1016/S0065-3454(05)35007-8.

Murray, L. (1998). The effects of group structure and rearing strategy on personality in chimpanzees Pan troglodytes at Chester, London ZSL and Twycross zoos. International Zoo Yearbook, 36, 97-108. https:// doi.org/10.1111/j.1748-1090.1998.tb02890.x.

Newman, M. E. (2004). Fast algorithm for detecting community structure in networks. Physical Review E, 69(6), 066133.

Pusey, A., Murray, C., Wallauer, W., Wilson, M., Wroblewski, E., \& Goodall, J. (2008). Severe aggression among female Pan troglodytes schweinfurthii at Gombe National Park, Tanzania. International Journal of Primatology, 29(4), 949-973. https://doi.org/10.1007/s10764-008-9281-6.

Rodrigues, M. A., \& Boeving, E. R. (2019). Comparative social grooming networks in captive chimpanzees and bonobos. Primates, 60(3), 191-202. https://doi.org/10.1007/s10329-018-0670-y.

Rose, P. E., \& Croft, D. P. (2015). The potential of social network analysis as a tool for the management of zoo animals. Animal Welfare, 24(2), 123-138.

Rox, A., van Vliet, A. H., Sterck, E. H. M., Langermans, J. A. M., \& Louwerse, A. L. (2019). Factors determining male introduction success and long-term stability in captive rhesus macaques. PLoS ONE, 14(7). https://doi.org/10.1371/journal.pone.0219972.

RStudio Team (2015). RStudio: Integrated development for R. RStudio, Inc. Boston, MA. http://www.rstudio.com/

Schel, A. M., Rawlings, B., Claidière, N., Wilke, C., Wathan, J., et al (2013). Network analysis of social changes in a captive chimpanzee community following the successful integration of two adult groups. American Journal of Primatology, 75(3), 254-266. https://doi.org/10.1002/ajp.22101.

Silk, J. B., Alberts, S. C., Altmann, J., Cheney, D. L., \& Seyfarth, R. M. (2012). Stability of partner choice among female baboons. Animal Behaviour, 83(6), 1511-1518. https://doi.org/10.1016/j.anbehav.2012.03.028.

Silk, J., Cheney, D., \& Seyfarth, R. (2013). A practical guide to the study of social relationships. Evolutionary Anthropology: Issues, News, and Reviews, 22(5), 213-225. https://doi.org/10.1002/evan.21367.

Silk, M. J., Finn, K. R., Porter, M. A., \& Pinter-Wollman, N. (2018). Can multilayer networks advance animal behavior research? Trends in Ecology and Evolution, 33(6), 376-378. https://doi.org/10.1016/j.tree.2018.03.008.

Smith-Aguilar, S. E., Aureli, F., Busia, L., Schaffner, C. M., \& Ramos-Fernandez, G. (2019). Using multiplex networks to capture the multidimensional nature of social structure. Primates, 60(3), 277-295. https://doi. org/10.1007/s10329-018-0686-3.

Suomi, S. J. (1997). Early determinants of behaviour: Evidence from primate studies. British Medical Bulletin, 53(1), 170-184. https://doi.org/10.1093/oxfordjournals.bmb.a011598.

Videan, E. N., \& Fritz, J. (2007). Effects of short- and long-term changes in spatial density on the social behavior of captive chimpanzees (Pan troglodytes). Applied Animal Behaviour Science, 102(1), 95-105. https://doi.org/10.1016/j.applanim.2006.03.011.

Whitehead, H., \& James, R. (2015). Generalized affiliation indices extract affiliations from social network data. Methods in Ecology and Evolution, 6(7), 836-844. https://doi.org/10.1111/2041-210X.12383.

Xia, D., Li, J., Garber, P. A., Sun, L., Zhu, Y., \& Sun, B. (2012). Grooming reciprocity in female tibetan macaques Macaca Thibetana. American Journal of Primatology, 74(6), 569-579. https://doi.org/10. 1002/ajp.21985.

Xia, D.-P., Li, J.-H., Garber, P. A., Matheson, M. D., Sun, B.-H., \& Zhu, Y. (2013). Grooming reciprocity in male Tibetan macaques. American Journal of Primatology, 75(10), 1009-1020. https://doi.org/10.1002/ajp.22165. 